\title{
Design and Analysis of A Bus Terminal Building
}

\author{
Bismi M Buhari ${ }^{1}$, Alan Varghese ${ }^{2}$, Bibin Babu ${ }^{3}$, Gokulkrishnan $\mathrm{R}^{4}$, Raveena R Nair ${ }^{5}$ \\ ${ }^{1}$ Asst. Professor, \\ 2345 Student \\ Civil Department, \\ Musaliar College of Engineering \& Technology, \\ Pathanamthitta, Kerala, India
}

\begin{abstract}
The design and analysis of bus terminal building is carried out. The manual designing of buildings include the design of foundation, design of column, beam, slab, stair etc using a set of procedures and building codes such as IS 456. Here the structural analysis using STAAD Pro V8i SS6 is carried out.
\end{abstract}

\section{Keywords: Deflection; Bending moment; Shear force;} Assembly building.

\section{INTRODUCTION}

In all spheres of human life buildings are always necessary to satisfy human need. This study mainly focuses on the analysis of an assembly building i.e Municipal bus terminal. Pandalam using STAAD Pro V8i SS6 and manual designing. In this study each structural part is analysed. The bending moment, deflection, shear force etc are analyzed.

\section{OBJECTIVES}

$>$ Generate structural frame work

$>$ Creating model using STAAD PRO V8i.

$>$ Carry out the structural analysis and manual design, Thus ensure the structural stability.

\section{BUILDING INFORMATIONS}

The proposed bus terminal is a two storey building. It consist of shops, office, staff room, toilet complex etc. there are two openings provided for the entry and exit of buses.

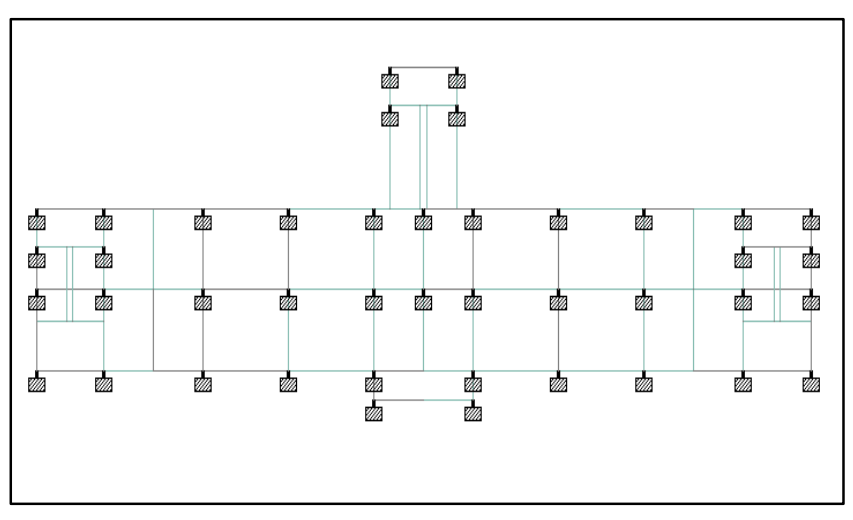

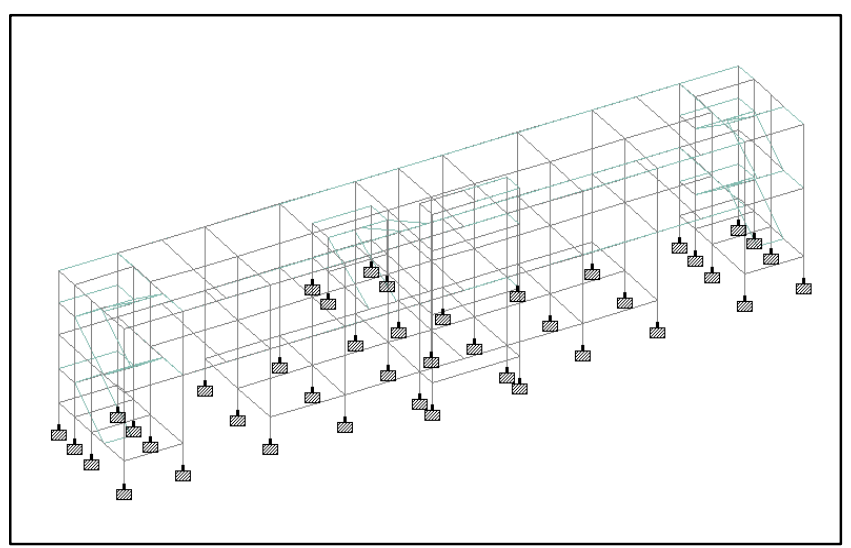

Fig.2 Structural analysis diagram

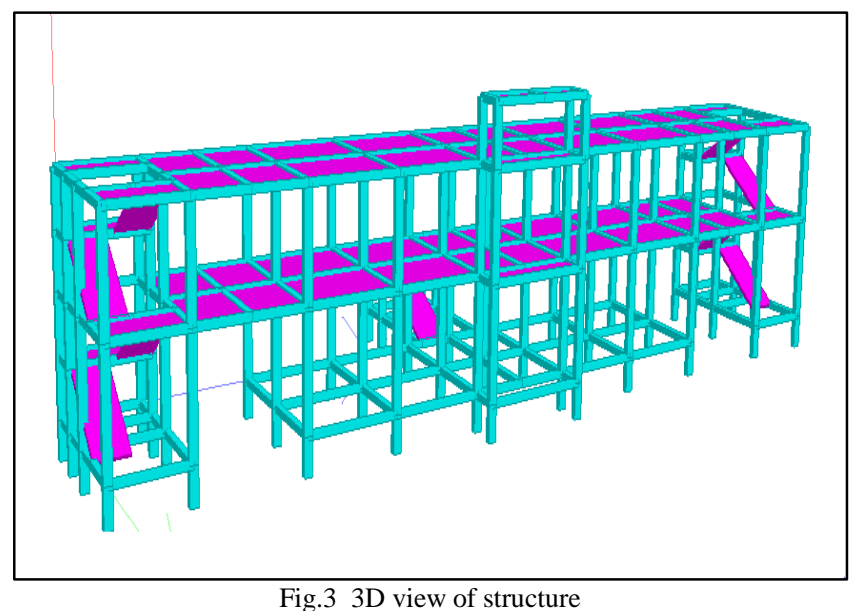

IV. SPECIFYING LOADS

These are self-weights of the structure to be designed. The dimensions of the cross section are to be assumed initially which enable to estimate the dead load from the known unit weights of the structure. The values of the unit weights of the materials are specified in IS 875:1987(Part-I). Dead load includes self-weight of columns, beams, slabs, brick walls, floor finish etc. The self-weight of the columns and beams were taken automatically by the software. The dead loads on the building are as follows. Here in STAAD the load given to the structure are Dead load ( Self weight and UDL due to brick of $18.6 \mathrm{KN} / \mathrm{m}$ ), Live load of $-3 \mathrm{KN} / \mathrm{m}^{2}$ and load combinations.

Fig.1 Plan of the analyzing structure 


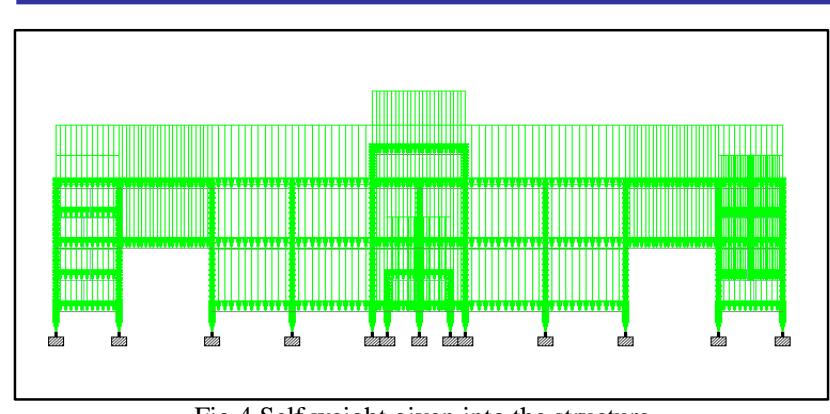

Fig.4 Self weight given into the structure

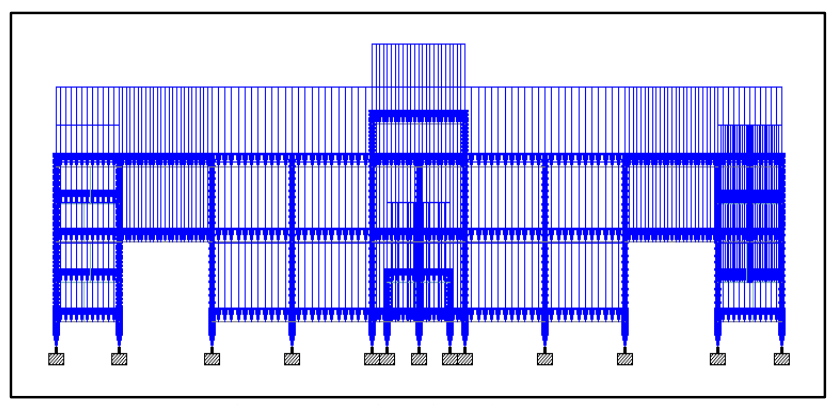

Fig. 5 UDL given into the structure

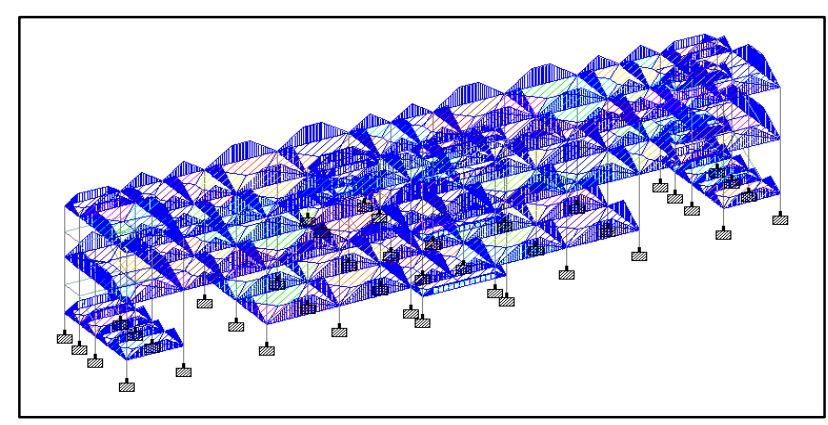

Fig.6 Live Load given into the structure

\section{MANUAL DESIGN AND STAAD ANALYSIS}

A. Design of pile cap

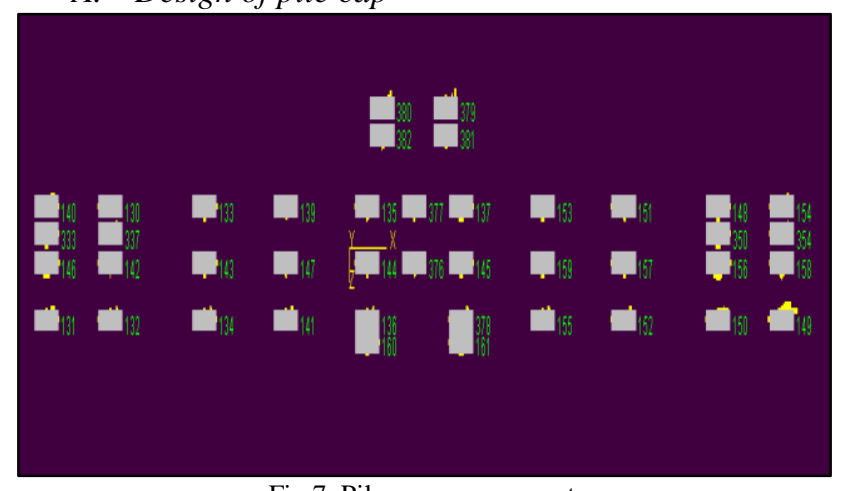

Datas

Number of piles

Pile Diameter

Fig.7 Pile cap arrangement.

Pix $2 \mathrm{hp}=2 \times 400=800 \mathrm{~m}$

Concrete Mix = M20

Steel Grade $\quad=\mathrm{Fe} 415$

Length of pile $\quad=23 \mathrm{~m}$.

Soil type is weak clayey soil

Assume unconfined compressive strength as $0.058 \mathrm{~N} / \mathrm{mm}^{2}$ i.e $\mathrm{UCS}=58 \mathrm{kN} / \mathrm{mm}^{2}$

Assume $\propto=0.9$

$$
\mathrm{C}_{\mathrm{u}}=\frac{58}{2}=29 \mathrm{kN} / \mathrm{m}^{2}
$$

Therefore, ultimate capacity of a single pile , $Q u$

$$
\begin{aligned}
& =\mathrm{C}_{\mathrm{u}} \mathrm{N}_{\mathrm{c}} \mathrm{A}_{\mathrm{p}}+\propto \mathrm{C}_{\mathrm{u}} \mathrm{A}_{\mathrm{s}} \\
& =965.726 \mathrm{kN}
\end{aligned}
$$

Spacing of pile $=2 \mathrm{~h}_{\mathrm{p}}=2 \times 500=1000 \mathrm{~mm}$

Dimension of column, $\mathrm{B} \times \mathrm{D}=0.45 \times 0.45 \mathrm{~m}$

Factored load $=1704.6 \mathrm{kN}$

Factored moment $=101.085 \mathrm{kN}$

Safe pile load capacity $=965.7 \mathrm{kN}$

\section{1) Pile Cap Dimension}

Length and breadth of pile cap $=1000+(2 \times 250)+$ $(150 \times 2)=1.8 \mathrm{~m}$

Depth of pile cap $=2 \mathrm{~h}_{\mathrm{p}}=1000 \mathrm{~mm}=1 \mathrm{~m}$

2) Check for pile load capacity

Self weight of pile cap $=(1.8 \times 1.8 \times 1 \times 25) \times 1.5=$ $121.5 k N$

Factored load from column, $\mathrm{Pu}=1704.6 \mathrm{kN}$

Total factored load $=121.5+1704.6=1826 \mathrm{kN}$

No. of pile $=4$

$\mathrm{y}$-ordinate of pile cap $=0.5 \mathrm{~m}$

$\mathrm{M}_{\mathrm{x}}=101.08 \mathrm{kNm}$

$$
\begin{gathered}
\text { Compressive load in pile about } \mathrm{X}-\text { axis }=\frac{\mathrm{P}_{\mathrm{u}}}{\mathrm{n}}+\frac{\mathrm{M}_{\mathrm{x}} \mathrm{y}}{\mathrm{y}^{2}} \\
=953.38 \mathrm{kN}
\end{gathered}
$$

Design working load $=\frac{953.38}{2}=635.58 \mathrm{kN}<965.7 \mathrm{kN}$, Hence safe.

3)

4)

$\mathrm{d}=410 \mathrm{~mm}$

$$
\begin{aligned}
& \text { Bending moment } \\
& \mathrm{M}_{\mathrm{u}}=953.38 \frac{(1-0.45)}{2}=262.18 \mathrm{kNm}
\end{aligned}
$$

Check for effective depth

$$
\begin{gathered}
\mathrm{M}_{\mathrm{u}}=0.138 \mathrm{df}_{\mathrm{ck}} \mathrm{d}^{2}=262.2 \mathrm{kNm} \\
\mathrm{d}^{2}=\frac{262.2 \times 10^{6}}{0.13 \times 25 \times 450}
\end{gathered}
$$

$$
\mathrm{d}_{\text {required }}<\mathrm{d}_{\text {provided }}
$$

Hence checked.

5) Check for punching shear

Punching shear at a distance $d / 2$ from face of column $=1704.6 \mathrm{kN}$

Perimeter of critical section $=2(1800+922)=5444 \mathrm{~mm}$

$$
\text { Punching shear }=\frac{1704.6 \times 10^{3}}{5444 \times 922}=0.399 \mathrm{~N} / \mathrm{mm}^{2}
$$

Allowable shear stress for $\mathrm{M}_{25}=0.25 \sqrt{\mathrm{f}_{\mathrm{ck}}}=1.25 \mathrm{~N} /$ $\mathrm{mm}^{2}>0.339 \mathrm{~N} / \mathrm{mm}^{2}$

Hence safe.

\section{6) Main reinforcement}

$\mathrm{Mu}=262.2 \mathrm{KNm}$

$$
\begin{gathered}
\mathrm{k}=\frac{\mathrm{M}_{\mathrm{u}}}{\mathrm{bd}^{2}}=0.17135 \\
\mathrm{~A}_{\text {st min }}=\frac{0.12}{100} \times 1800 \times 922=1991.52 \mathrm{~mm}^{2}
\end{gathered}
$$




$$
\text { No. of bars }=\frac{1991.52}{201.1}=9.905 \approx 10 \text { bars }
$$

Therefore provide $16 \mathrm{~mm} \emptyset, 10$ bars at bottom.

Reinforcement at top,

Minimum $\mathrm{A}_{\mathrm{st}}=1991.52 \mathrm{~mm}^{2}$

Therefore provide $16 \mathrm{~mm} \emptyset, 10$ bars at top.

\section{7) Check for one way shear}

Maximum shear force at facing of column $=958.38 \mathrm{kN}$

Shear stress $=0.577 \mathrm{~N} / \mathrm{mm}^{2}$

For $\mathrm{P}_{\mathrm{t}}=0.2 \%$,

$$
\tau_{\mathrm{c}} \text { from IS456 }=0.33 \mathrm{~N} / \mathrm{mm}^{2}
$$

Shear to be carried by stirrups,

$\mathrm{V}_{\mathrm{u}}=(0.577-0.33) \times 1800 \times 922 \times 10^{-3}=409.92 \mathrm{kN}$

$$
\frac{\mathrm{V}_{\mathrm{u}}}{\mathrm{d}}=4.446 \mathrm{kN} / \mathrm{m}
$$

Provide $10 \mathrm{~mm} \emptyset, 4$ legged stirrups at $120 \mathrm{~mm} \mathrm{c} / \mathrm{c}$ spacing.

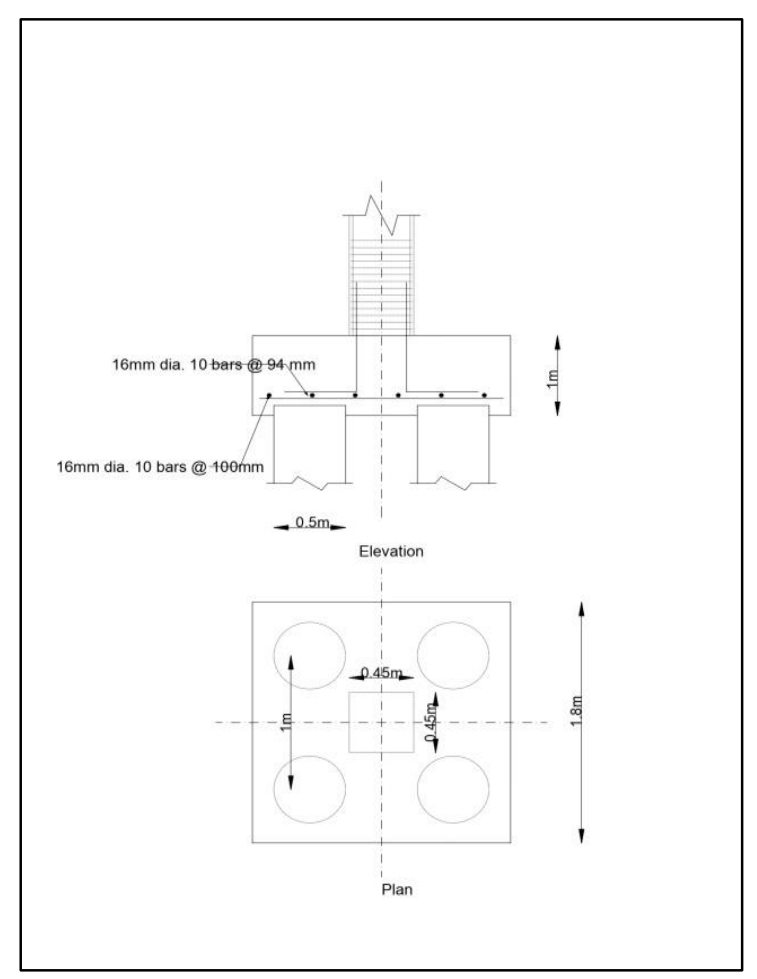

Fig. 8 Pile cap detailing

$$
\text { B. }
$$

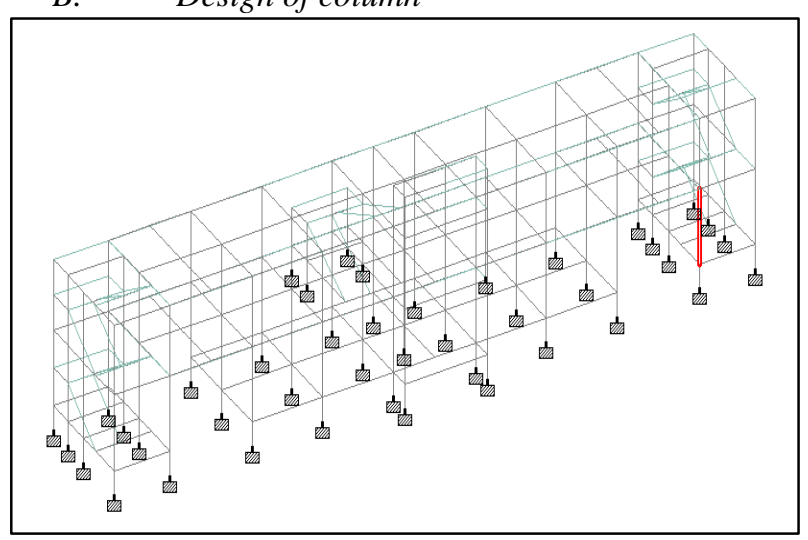

Fig. 9 Column no. 380 in the structure

\section{Column no.380}

Grade of Concrete $=$ M30

Grade of Steel $=$ Fe415

Characteristic compressive strength of concrete,

$\mathrm{f}_{\mathrm{ck}}\left(\mathrm{N} / \mathrm{mm}^{2}\right)=30$

Characteristic yield strength of steel,

$\mathrm{f}_{\mathrm{y}}\left(\mathrm{N} / \mathrm{mm}^{2}\right)=415$

Unit weight of concrete, $\gamma_{\mathrm{c}}\left(\mathrm{kN} / \mathrm{m}^{3}\right)=25$

Partial safety factor for concrete $=1.5$

Exposure condition $=$ Mild

Nominal Cover to exposure condition $(\mathrm{mm})=40$

Assumed effective cover all around, d' $(\mathrm{mm})=40$

\section{1) Dimensions of the Column}

Breadth of the column B $(\mathrm{mm})=450$

Depth of the Column D $(\mathrm{mm})=450$

Least lateral dimension $=450 \mathrm{~mm}$

\section{2) Design Factors}

Factored load, $\mathrm{Pu}=2208 \mathrm{kN}$

Factored moment acting parallel to the larger dimension, Mux $=36 \mathrm{kNm}$

Factored moment acting parallel to the shorter dimension, Muy $=48 \mathrm{kNm}$

Assume percentage of steel $=1.5 \%$

\section{3) Moment in $X-X$ Direction}

$\frac{\mathrm{d}^{\prime}}{\mathrm{D}}=\frac{40}{450}=0.09 \approx 0.1$

$\frac{\mathrm{Pu}}{\mathrm{fckbD}}=\frac{2208 \times 1000}{25 \times 450 \times 450}=0.436 \approx 0.44$

$\frac{\mathrm{p}}{\mathrm{fck}}=\frac{1.5}{25}=0.06$

$\frac{\mathrm{Mu}}{\mathrm{fckbD}^{2}}=0.07$

Mux $^{\prime}=0.07 \times 25 \times 450 \times 450^{2}$

$=159.46 \times 10^{6} \mathrm{Nmm}$

$$
\begin{aligned}
& \text { 4) Moment in } Y-Y \text { Direction } \\
& \frac{\mathrm{d}^{\prime}}{\mathrm{D}}=\frac{40}{450}=0.09 \approx 0.1 \\
& \frac{\mathrm{Pu}}{\mathrm{fckbD}}=\frac{2208 \times 1000}{25 \times 450 \times 450}=0.436 \approx 0.44 \\
& \frac{\mathrm{p}}{\mathrm{fck}}=\frac{1.5}{25}=0.06 \\
& \frac{\mathrm{Mu}}{\mathrm{fckbD}^{2}}=0.07 \\
& \text { Muy' }^{\prime}=0.07 \times 25 \times 450 \times 450^{2} \\
& =159.46 \times 10^{6} \mathrm{Nmm}
\end{aligned}
$$

\section{5) Calculation of $P_{u z}$}

$\mathrm{p}=1.5 \%$

$\frac{100 \mathrm{~A}_{\mathrm{sc}}}{\mathrm{bD}}=1.5$

$A_{\text {sc }}=\frac{1.5 \times 450 \times 450}{100}=3037.5 \mathrm{~mm}^{2}$

$A_{c}=A_{g}-A_{s c}=199462.5 m^{2}$ 
$\mathrm{P}_{\mathrm{uz}}=0.45 \mathrm{f}_{\mathrm{ck}} \mathrm{A}_{\mathrm{c}}+0.75 \mathrm{f}_{\mathrm{y}} \mathrm{A}_{\mathrm{sc}}=3189.37 \mathrm{kN}$

$\frac{\mathrm{Pu}}{\mathrm{Puz}}=\frac{2208.36}{3189.37}=0.69$

From IS 456, value of $\propto_{n}=1.82$

Check

$\left(\frac{\operatorname{Mux}}{\operatorname{Mux}^{\prime}}\right)^{\alpha_{\mathrm{n}}}+\left(\frac{\text { Muy }}{\text { Muy' }^{\prime}}\right)^{\alpha_{\mathrm{n}}} \leq 1$ $\left(\frac{36.03 \times 10^{6}}{159.46 \times 10^{6}}\right)^{1.82}+\left(\frac{47.95 \times 10^{6}}{159.46 \times 10^{6}}\right)^{1.82}=0.1789$ $<1$

Therefore, the column is safe. $1.5 \%$ of steel is sufficient.

$\mathrm{p}=1.5 \%$

6) Calculation of reinforcement

$\frac{100 \mathrm{~A}_{\text {st }}}{\mathrm{bD}}=1.5$

$\mathrm{A}_{\text {st }}=\frac{1.5 \times 450 \times 450}{100}=3037.5 \mathrm{~mm}^{2}$

Assume $16 \emptyset \mathrm{mm}$ bars.

No. of bars $=\frac{A_{\mathrm{st}}}{\mathrm{A} \phi_{\varnothing}}=15.11 \approx 16$ bars

Provide 16 bars of $16 \mathrm{~mm}$ diameter.

The STAAD diagrams are the following,

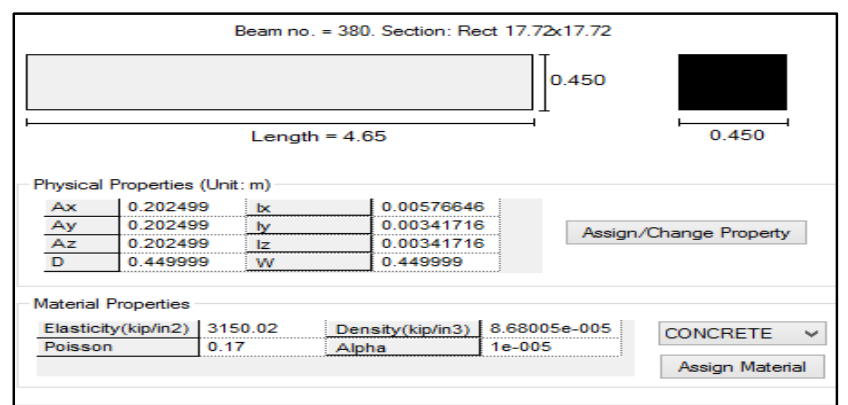

Fig.10 Dimensional details of Member 380 in the structure

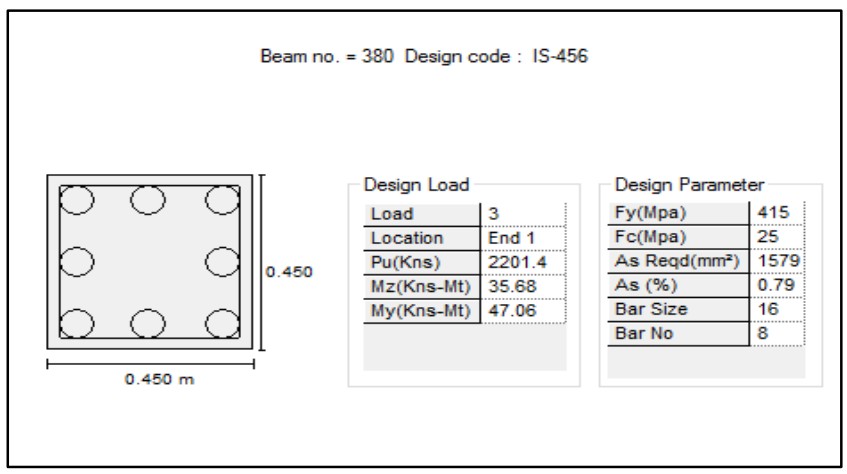

Fig.11 Reinforcement details of Member 380 in the structure

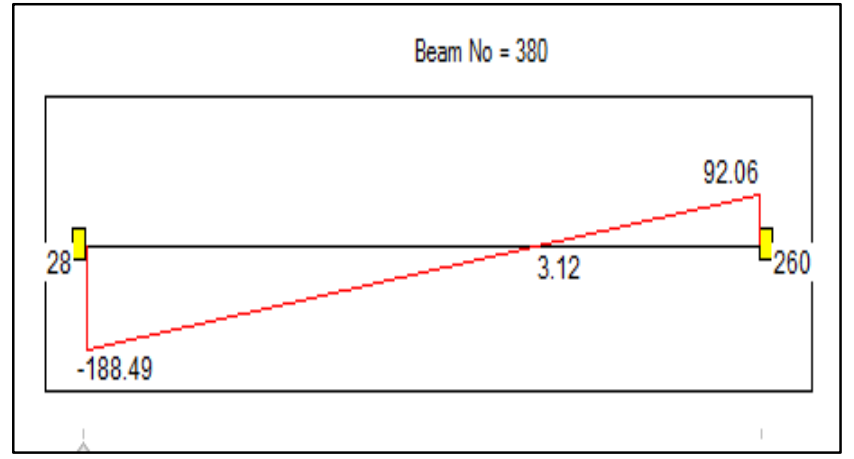

Fig.12 Shear bending diagram of Member 380 in the structure

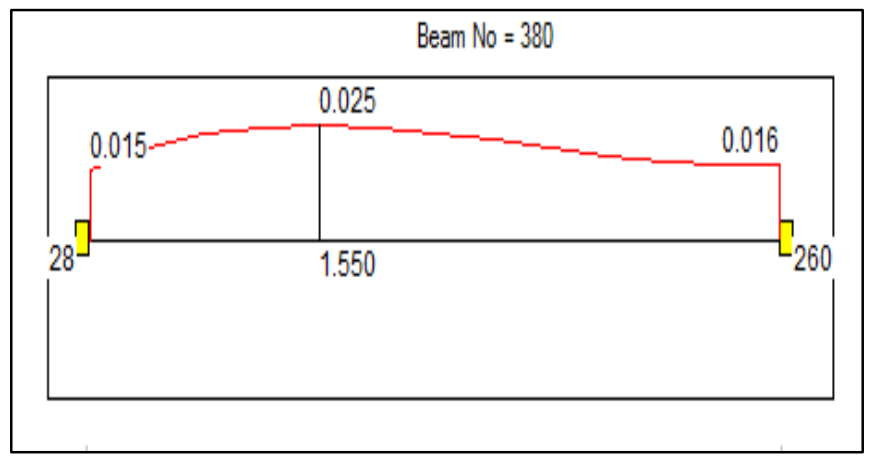

Fig.13 Deflection diagram of Member 380 in the structure

C.

Design of beam

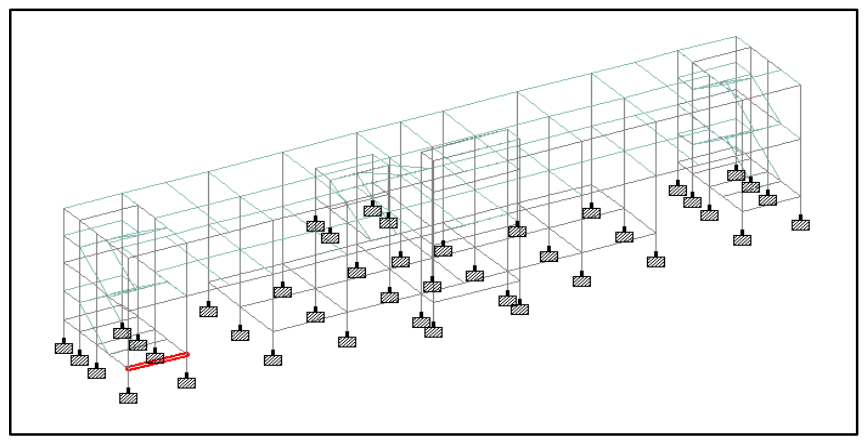

Beam no. 4

Fig. 14 Beam no. 4 in the structure

Clear $\operatorname{span}=4.2 \mathrm{~m}$

Live load $=12 \mathrm{kN} / \mathrm{m}$

$\mathrm{f}_{\mathrm{ck}}=25 \mathrm{~N} / \mathrm{mm}^{2}$

$\mathrm{f}_{\mathrm{y}}=415 \mathrm{~N} / \mathrm{mm}^{2}$

$$
\begin{aligned}
& \text { 1) Calculation of }\left(\frac{x_{u}}{d_{\text {lim }}}\right) \\
& \frac{\mathrm{x}_{\mathrm{u}}}{\mathrm{d}}=\frac{700}{1100+0.87 \mathrm{f}_{\mathrm{y}}}=0.48
\end{aligned}
$$

Assumptions of cross sectional dimensions

$$
\begin{gathered}
\frac{\mathrm{l}}{\mathrm{d}}=15 \\
\frac{4200}{15}=\mathrm{d}
\end{gathered}
$$

$\mathrm{d}=280 \mathrm{~mm} \approx 300 \mathrm{~mm}$

$\mathrm{D}=\mathrm{d}+50=350 \mathrm{~mm}$

$$
\frac{\mathrm{D}}{\mathrm{b}}=2
$$


$\mathrm{b}=175 \mathrm{~mm} \approx 200 \mathrm{~mm}$

\section{2) Load calculation}

Self weight $=\mathrm{b} \times \mathrm{D} \times \Upsilon_{\text {con }}=1.75 \mathrm{~N} / \mathrm{mm}$

Dead load $=1.75 \mathrm{~N} / \mathrm{mm}$

Design load $=(\mathrm{DL}+\mathrm{LL}) \Upsilon_{\mathrm{con}}=20.63 \mathrm{~N} / \mathrm{mm}$

3) Design bending moment

$$
\mathrm{BM}=\frac{\mathrm{Wl}^{2}}{8}=52.22 \mathrm{kNm}
$$

4) Calculation of limiting value of moment

$$
\begin{gathered}
\left(\mathrm{M}_{\mathrm{u}}\right)_{\lim }=0.36 \frac{\mathrm{x}_{\mathrm{u}}}{\mathrm{d}}\left[1-0.42 \frac{\mathrm{x}_{\mathrm{u}}}{\mathrm{d}}\right] \mathrm{f}_{\mathrm{ck}} \mathrm{bd}^{2} \\
=62.08 \mathrm{kNm}
\end{gathered}
$$

$\mathrm{BM}<\left(\mathrm{M}_{\mathrm{u}}\right)_{\text {lim }}$

Therefore under reinforced.

5) Reinforcement calculation

$\mathrm{M}_{\mathrm{u}}=0.87 \mathrm{f}_{\mathrm{y}} \mathrm{A}_{\mathrm{st}} \mathrm{d}\left[1-\frac{\mathrm{f}_{\mathrm{y}} \mathrm{A}_{\mathrm{st}}}{\mathrm{f}_{\mathrm{ck}} \mathrm{bd}}\right]$

$\mathrm{A}_{\mathrm{st}}=572.60 \mathrm{~mm}^{2}$

Assume $16 \mathrm{~mm} \emptyset$ bars.

No. of bars $=\frac{\text { total area }}{\text { area of one bar }}=2.84 \approx 4$ bars

6) Design for shear

$$
\begin{gathered}
\mathrm{V}_{\mathrm{u}}=\frac{\mathrm{Wl}_{\mathrm{e}}}{2}=46417.5 \mathrm{~N} / \mathrm{mm}^{2} \\
\tau_{\mathrm{v}}=\frac{\mathrm{V}_{\mathrm{u}}}{\mathrm{bd}}=0.77 \mathrm{~N} / \mathrm{mm}^{2}
\end{gathered}
$$

7) Design shear strength

Percentage of steel $=\frac{100 \mathrm{~A}_{\mathrm{st}}}{\mathrm{bd}}=1.0048$

$\tau_{\mathrm{c}}=0.64 \mathrm{~N} / \mathrm{mm}^{2}$

$\tau_{\mathrm{c} \max }=3.1 \mathrm{~N} / \mathrm{mm}^{2}$

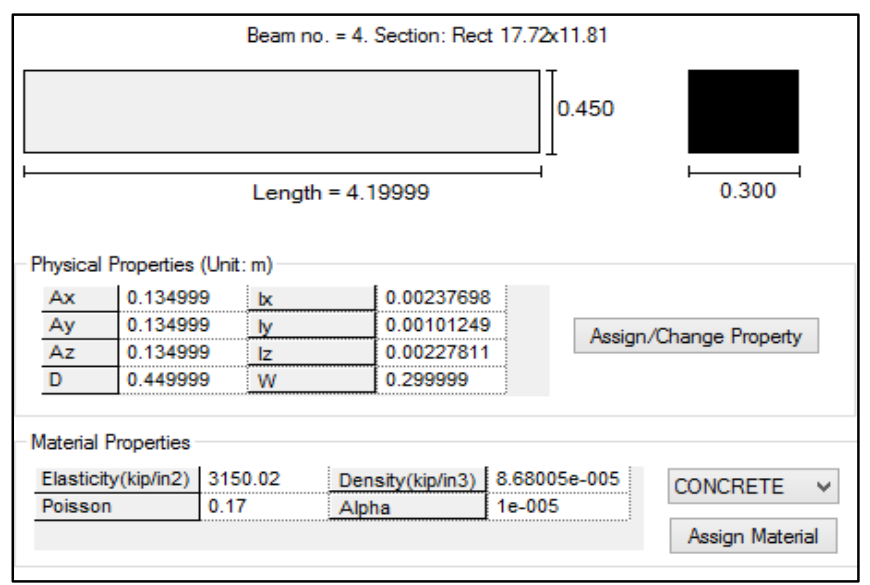

Fig.15 Dimensional details of Member 4 in the structure

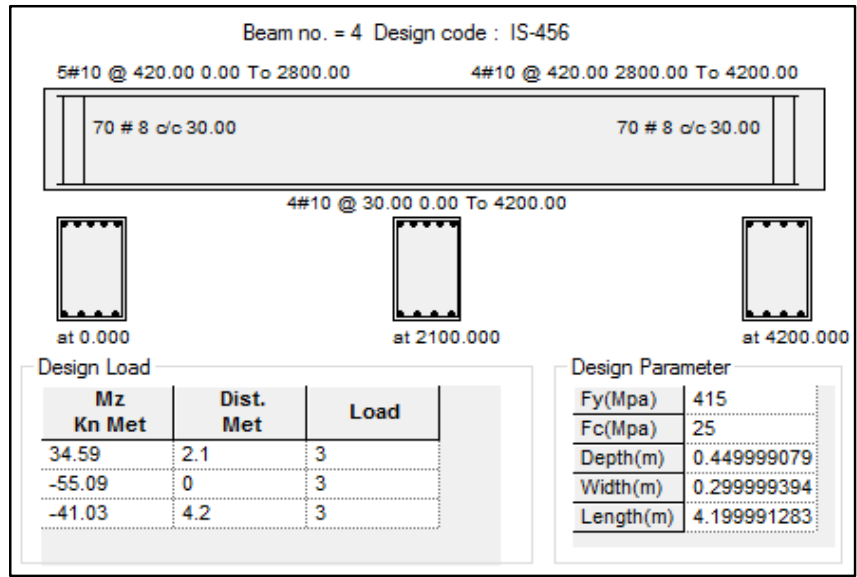

Fig.16 Reinforcement details of Member 4 in the structure

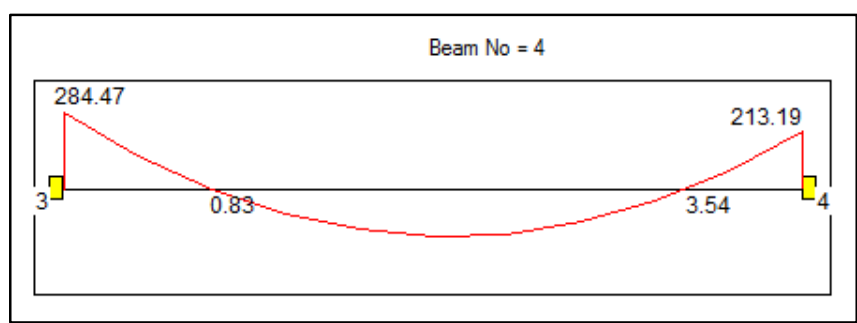

Fig.17 Shear bending diagram of Member 4 in the structure

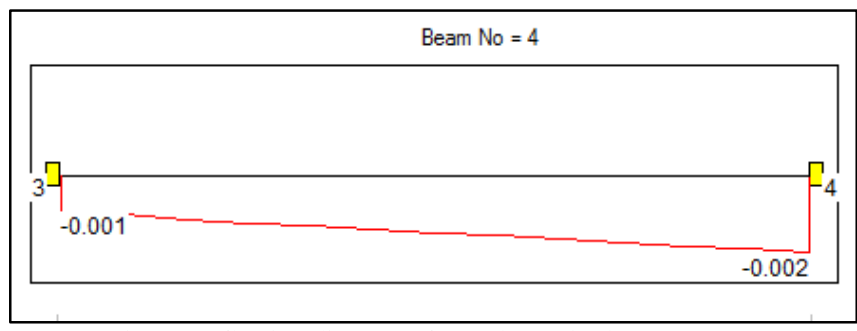

Fig.18 Deflection diagram of Member 4 in the structure

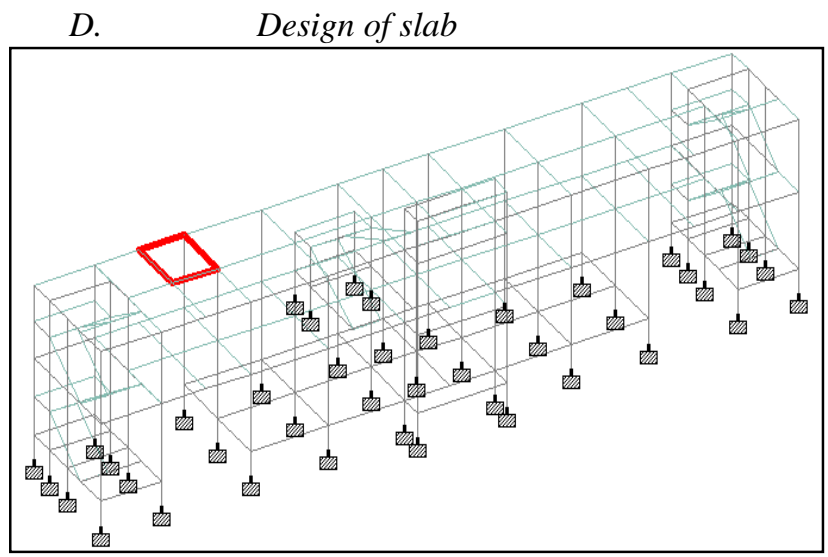

Fig.19 Slab 707 in the structure.

Slab no. 707

Size $4.2 \times 2.4 \mathrm{~m}$

M25 and Fe415 are used.

$$
\begin{aligned}
& \text { 1) Type of slab } \\
& \frac{\mathrm{l}_{\mathrm{y}}}{\mathrm{l}_{\mathrm{x}}}=\frac{4.2}{2.4}=1.75
\end{aligned}
$$


Therefore it is a two way slab.

$$
\begin{aligned}
& \text { 2) Depth of slab } \\
& \frac{\mathrm{l}}{\mathrm{d}}=20 \\
& \mathrm{~d}=\frac{4200}{20 \times 1.5}=136.66 \approx 150 \mathrm{~mm} \\
& \text { Effective span }=\text { clear } \text { span }+\mathrm{d} \\
& =4200+150 \\
& =4350 \mathrm{~mm}
\end{aligned}
$$

Depth ,D $=150+20=170 \mathrm{~mm}$

$$
\text { 3) Design load }
$$

Design load $=1.5(\mathrm{DL}+\mathrm{LL})$

$$
\begin{aligned}
& =1.5(4.25+3) \\
& =10.875 \mathrm{kN} / \mathrm{m}^{2}
\end{aligned}
$$

4) $\quad M$ along $X$ and $Y$

$\mathrm{T}$ he slab is restrained,

From IS 456, $\frac{l_{y}}{l_{x}}=1.75$

$\propto_{x=0.1} \propto_{y=0.056}$

$\propto_{y=0.056}$

$$
\begin{aligned}
\mathrm{Mx} & =\propto_{\mathrm{x}} \mathrm{wl}_{\mathrm{x}}{ }^{2} \\
& =0.1 \times 10.875 \times 4.350^{2} \\
& =20.577 \mathrm{kNm} \\
\mathrm{My} & =\propto_{\mathrm{y}} \mathrm{wl}_{\mathrm{y}}{ }^{2} \\
& =0.056 \times 10.875 \times 4.35^{2} \\
& =11.523 \mathrm{kNm}
\end{aligned}
$$

Shear Force $=\frac{\mathrm{wl}}{2}$

$$
\begin{gathered}
=\frac{10.875 \times 4.35}{2} \\
=23.65 \mathrm{kN}
\end{gathered}
$$

$$
\begin{aligned}
\text { 5) } & =\sqrt{\frac{\mathrm{M}_{\mathrm{x}}}{0.138 \mathrm{f}_{\mathrm{ck}} \mathrm{b}}} \\
& =\sqrt{\frac{20.577 \times 10^{6}}{0.138 \times 25 \times 1000}}
\end{aligned}
$$

$=77.229 \mathrm{~mm}$

Hence, $\mathrm{d}_{\text {required }}<\mathrm{d}_{\text {provided }}$.

6) Calculation of Ast (short san)

$\left(\mathrm{M}_{\mathrm{u}}\right)_{\lim }=0.87 \mathrm{f}_{\mathrm{y}} \mathrm{A}_{\mathrm{st}} \mathrm{d}\left(1-\frac{\mathrm{A}_{\text {stf }} \mathrm{y}}{\mathrm{bd} \times \mathrm{f}_{\mathrm{ck}}}\right)$

$\mathrm{A}_{\text {st }}=397.27 \mathrm{~mm}^{2}$

Assume $10 \mathrm{~mm}$ dia bars.

$$
\begin{aligned}
\mathrm{S} & =\frac{1000 \mathrm{~A}_{\emptyset}}{\mathrm{A}_{\mathrm{St}}} \\
& =197 \mathrm{~mm} \\
& \approx 195 \mathrm{~mm}
\end{aligned}
$$

Provide $10 \mathrm{~mm}$ dia bars at $195 \mathrm{~mm} \mathrm{c} / \mathrm{c}$ along shorter span.

\section{7) Calculation of Ast (long san)}

$\mathrm{d}^{\prime}=$ effective depth - (0.5 dia of mai bar

$$
=140 \mathrm{~mm}
$$$$
+0.5 \text { dia of main bar) }
$$

$\left(M_{u}\right)_{\lim }=0.87 f_{y} A_{s t} d^{\prime}\left(1-\frac{A_{s t f y}}{b d \times f_{c k}}\right)$

$$
\begin{aligned}
\mathrm{A}_{\mathrm{st}} & =234.422 \mathrm{~mm}^{2} \\
\mathrm{~s} & =\frac{1000 \mathrm{~A}_{\emptyset}}{\mathrm{A}_{\mathrm{st}}} \\
& =334.994 \mathrm{~mm} \\
& \approx 330 \mathrm{~mm}
\end{aligned}
$$

Provide $10 \mathrm{~mm}$ dia bars at $330 \mathrm{~mm} \mathrm{c} / \mathrm{c}$.

$$
\begin{aligned}
& \text { 8) } \begin{aligned}
\left(\frac{\mathrm{l}}{\mathrm{d}}\right)_{\min } & =\left(\frac{\mathrm{l}}{\mathrm{d}}\right)_{\text {basic }} \times \mathrm{k}_{\mathrm{t}} \times \mathrm{k}_{\mathrm{f}} \times \mathrm{k}_{\mathrm{s}} \\
= & 20 \times 1.7 \\
= & 34 \\
\left(\frac{\mathrm{l}}{\mathrm{d}}\right)_{\text {max }}= & \frac{4350}{150} \\
= & 29<34
\end{aligned}
\end{aligned}
$$

Therefore the slab is safe.

$$
\begin{aligned}
& \text { 9) Checkfor shear } \\
& \mathrm{V}_{\mathrm{u}}=\frac{\mathrm{wl}}{2}=23.65 \mathrm{kN} \\
& \tau_{\mathrm{u}}=\frac{\mathrm{V}_{\mathrm{u}}}{\mathrm{bd}}=\frac{23.65 \times 10^{3}}{1000 \times 150}
\end{aligned}
$$

Percentage of steel $=\frac{100 A_{s t}}{b d}=0.264$.

E. Design of stairs

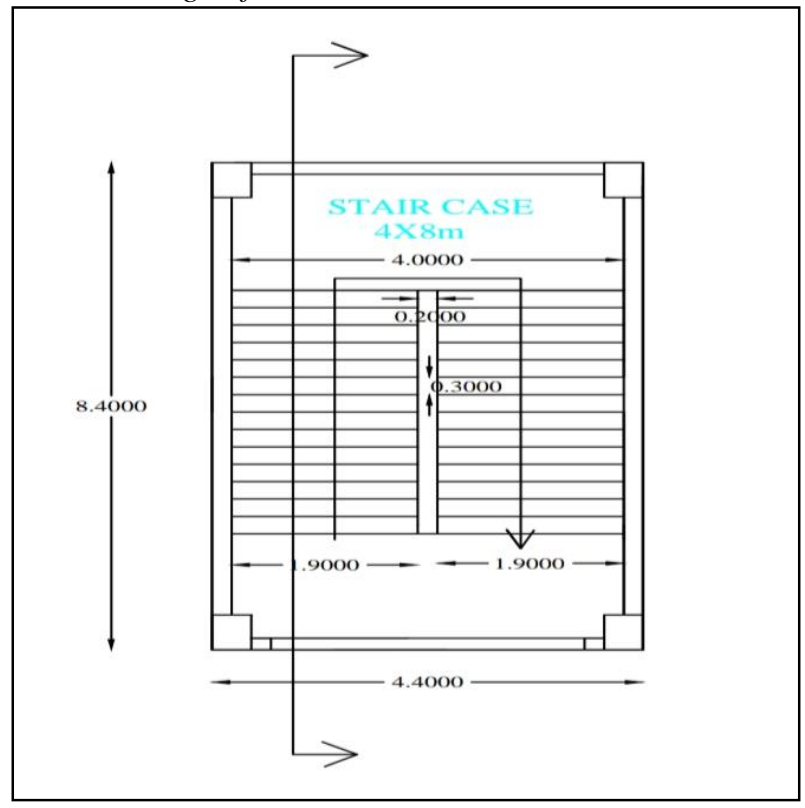

Fig.20 Stair case details.

Material Constants

Concrete, $\mathrm{f}_{\mathrm{ck}}=25 \mathrm{~N} / \mathrm{mm}^{2}$

Steel, $\mathrm{f}_{\mathrm{y}}=415 \mathrm{~N} / \mathrm{mm}^{2}$

\section{1) Dimensioning}

Height of each flight $=\frac{4.35}{2}=2.175 \mathrm{~m}$

Let the tread of steps be $300 \mathrm{~mm}$.

Rise of stair $=0.145 \mathrm{~m}$

Width of stair $=1.9 \mathrm{~m}$ 
Effective span, $1_{\mathrm{e}}=8.4 \mathrm{~m}$

Let the thickness of waist slab be $150 \mathrm{~mm}$.

Use $12 \mathrm{~mm} \phi$ bars.

Assume, clear cover $=25 \mathrm{~mm}$

Effective depth $=119 \mathrm{~mm}$

\section{2) Loads on landing slab}

Self weight of Slab $=0.15 \times 25=3.75 \mathrm{kN} / \mathrm{m}^{2}$

Finishes $=1.25 \mathrm{kN} / \mathrm{m}^{2}$

Live Load on Slab $=4 \mathrm{kN} / \mathrm{m}^{2}$

Total $=9 \mathrm{kN} / \mathrm{m}^{2}$

Factored load $=1.5 \times 9=13.50 \mathrm{kN} / \mathrm{m}^{2}$

\section{3) Loads on waist slab}

Dead load of waist slab $=\frac{\text { thickness of waist slab } \times 25 \times \sqrt{\mathrm{R}^{2}+\mathrm{T}^{2}}}{\mathrm{~T}}$

$$
\begin{aligned}
= & \frac{0.15 \times 25 \times \sqrt{0.145^{2}+0.3^{2}}}{0.3} \\
= & 4.165 \mathrm{kN} / \mathrm{m}^{2}
\end{aligned}
$$

Self weight of step $=0.5 \times \mathrm{R} \times 25=1.8125 \mathrm{kN} / \mathrm{m}^{2}$

Floor finish $=1.25 \mathrm{kN} / \mathrm{m}^{2}$

As per IS: 875(Part 2),1987, Table-1.

Live load $=4 \mathrm{kN} / \mathrm{m}^{2}$

Total service load $=11.228 \mathrm{kN} / \mathrm{m}^{2}$

Consider $1 \mathrm{~m}$ width of waist slab.

Total service load $/ \mathrm{m}$ run $=11.228 \times 1$

$$
=11.228 \mathrm{kN} / \mathrm{m}
$$

Factored load, $\mathrm{Wu}=1.5 \times 11.228=16.842 \mathrm{kN} / \mathrm{m}$

As per the UDL of the landing slab and waist slab,

Reaction $\mathrm{R}_{\mathrm{A}}=45.414 \mathrm{kN}$

Reaction $\mathrm{R}_{\mathrm{B}}=49.626 \mathrm{kN}$

\section{4) Bending moment}

To get maximum Bending Moment, take Shear Force at $\mathrm{x}$ distance from support $B=0$. Thus obtained $X$ is $2.95 \mathrm{~m}$.

Maximum moment at $\mathrm{X}=2.95 \mathrm{~m}$,

$\mathrm{Mu}=73.1187 \mathrm{kNm}$

\section{5) Reinforcement calculations.}

$\frac{\mathrm{M}_{\mathrm{u}}}{\mathrm{bd}^{2}}=5.16 \mathrm{~N} / \mathrm{mm}^{2}$

Percentage of steel, $\mathrm{p}_{\mathrm{t}}=1.19 \%$

(From SP16, Table 3)

Therefore, Ast $=\frac{\mathrm{p}_{\mathrm{t}} \mathrm{bd}}{100}=1416.1 \mathrm{~mm}^{2}$

Minimum steel $=0.12 \%$ cross sectional area

$$
=142.8 \mathrm{~mm}^{2}
$$

Use $12 \mathrm{~mm} \varnothing$ bars,

Spacing $=\frac{1000 \mathrm{~A}_{\emptyset}}{\mathrm{A}_{\mathrm{st}}}=79.82 \mathrm{~mm}$

Provide $12 \mathrm{~mm} \varnothing$ bars at $80 \mathrm{~mm} \mathrm{c} / \mathrm{c}$.

Maximum Spacing $=3 \mathrm{~d}=3 \times 119$

$$
=357 \mathrm{~mm} \text { or } 300 \mathrm{~mm} \text { (less) }
$$

Hence, provide reinforcement of $12 \mathrm{~mm} \varnothing$ bars at $80 \mathrm{~mm}$ c/c

Distribution steel $=0.12 \%$ cross sectional area $=142.8 \mathrm{~mm}^{2}$

Provide $8 \mathrm{~mm} \emptyset$ bars.

Spacing $=\frac{1000 \mathrm{~A}_{\emptyset}}{\mathrm{A}_{\mathrm{st}}}=351.82 \mathrm{~mm}$

Maximum Spacing $=4 \mathrm{~d}=476 \mathrm{~mm}$
Hence, Provide $8 \mathrm{~mm}$ diameter bars at $350 \mathrm{~mm} \mathrm{c} / \mathrm{c}$.

\section{6) Check for shear}

(As per IS 456:2000, Clause 40)

Maximum Shear force, $V=49.626 \mathrm{kN}$

Nominal shear stress, $\tau_{\mathrm{v}}=\frac{\mathrm{V}_{\mathrm{u}}}{\mathrm{bd}}=0.417 \mathrm{~N} / \mathrm{mm}^{2}$

Max. value of shear stress, $\tau_{c} \max =3.1 \mathrm{~N} / \mathrm{mm}^{2}$

To get design shear strength of concrete,

$\frac{100 \mathrm{As}}{\mathrm{bd}^{2}}=0.09<0.15$

From IS 456: 2000, Table - 19

$\tau_{\mathrm{c}}=0.64 \mathrm{~N} / \mathrm{mm}^{2}, \tau_{\mathrm{v}}<\tau_{\mathrm{c}}<\tau_{\mathrm{c} \max }$

So, shear reinforcement is not required.

\section{VI . $\quad 3 D$ MODEL OF BUS TERMINAL}

The 3D model of the bus terminal building is prepared. The model is prepared as per the plan and other details.

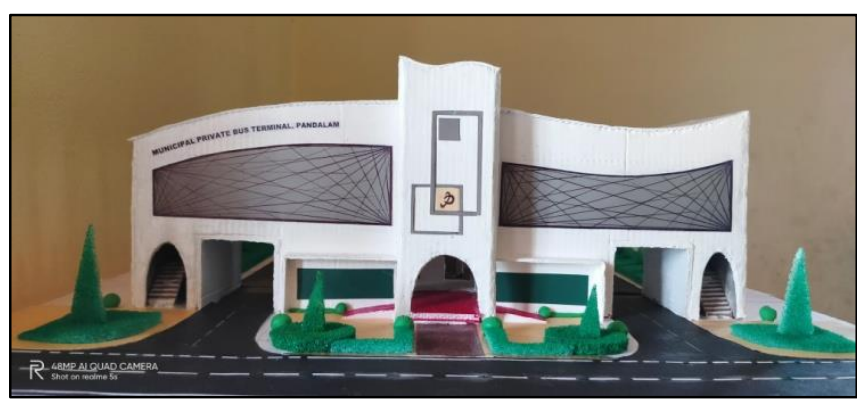

Fig.21 Elevation of model.

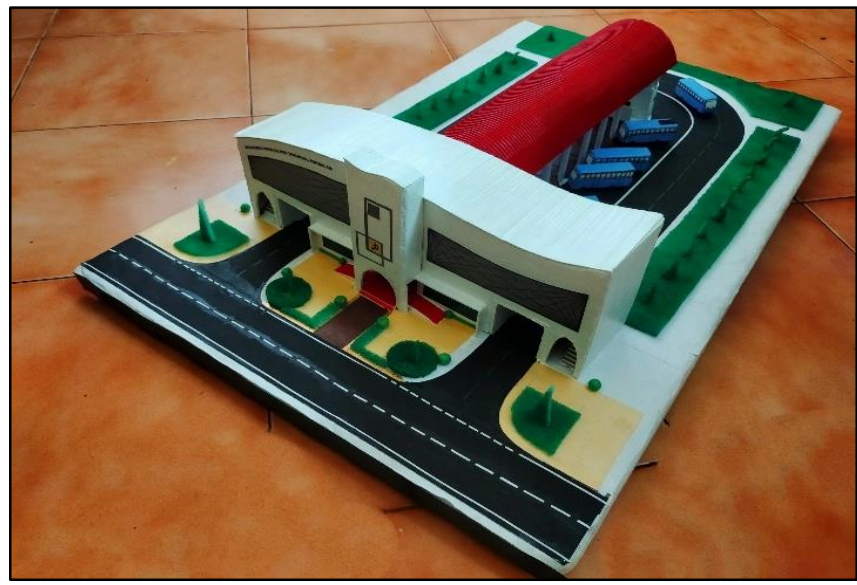

Fig. 22 Model of bus terminal

\section{VII . CONCLUSION}

- The structure is completely analysed using STAAD PRO v8i.

- The structural components of the building are safe in shear and flexure.

- Amount of steel provided for the structure is economic.

- Proposed sizes of the elements can be used in the structure. 


\section{REFERENCES}

[1]. Mahesh Ram Patel; R.C. Singh;' Analysis of a tall structure using staad pro providing different wind intensities as per 875 part-iii”, IJERST , International Journal of Engineering Sciences \& Research Technology, May 2017.

[2]. Aman; Manjunath Nalwadgi ; Vishal T; Gajendra "Analysis and design of multistorey building by using STAAD Pro IS 456-2000",International Research Journal of Engineering and Technology (IRJET), Volume: 03 Issue: 06, June-2016.

[3]. Anoop A ; Fousiya Hussian; Neeraja R; Rahul Chandran; Shabina S; Varsha S; Anjali A;" Planning, analysis and design of multi storied building by staad.pro.v8i", International Journal of Scientific \& Engineering Research, Volume 7, Issue 4, April-2016.

[4]. IS: 875 (Part 1) 1987( Dead Load )

[5]. IS: 875 (Part 2) 1987( Live Load ).

[6]. Design Aids For Reinforced Concrete to IS: 456-1978

[7]. IS. 456: 2000 